\title{
Editorial
}

\section{Nonlinear Dynamics in Applied Sciences Systems: Advances and Perspectives}

\author{
Luca Guerrini, ${ }^{1}$ Carlo Bianca, ${ }^{2}$ Massimiliano Ferrara, ${ }^{3}$ and Constantin Udriste ${ }^{4}$ \\ ${ }^{1}$ Department of Management, Polytechnic University of Marche, 60121 Ancona, Italy \\ ${ }^{2}$ Dipartimento di Scienze Matematiche, Politecnico, Corso Duca degli Abruzzi 24, 10129 Torino, Italy \\ ${ }^{3}$ Department of Law and Economics, University Mediterranea of Reggio Calabria and CRIOS University Bocconi of Milan, \\ Via dei Bianchi 2, 89127 Reggio Calabria, Italy \\ ${ }^{4}$ Department of Mathematics-Informatics, University Politehnica of Bucharest, Splaiul Independentei 313, 060042 Bucharest, Romania
}

Correspondence should be addressed to Luca Guerrini; luca.guerrini@univpm.it

Received 4 September 2013; Accepted 4 September 2013; Published 25 May 2014

Copyright (C) 2014 Luca Guerrini et al. This is an open access article distributed under the Creative Commons Attribution License, which permits unrestricted use, distribution, and reproduction in any medium, provided the original work is properly cited.

This editorial provides a brief review of some concepts related to the subject of the papers published in this special issue devoted to the onset of nonlinear dynamics in systems of the applied sciences. Nonlinear dynamics is currently an active and fashionable discipline that is having a profound effect on a wide variety of fields, including populations dynamics, physics, biology, economics, and sociology.

The origin of nonlinear dynamics is related to the gravitational three-body problem [1], which attempts to calculate the orbit of a planet around the sun in presence of two celestial bodies (planets or moon). In particular, the presence of a third celestial body can influence the dynamics of the plane and produce highly irregular dynamics (chaotic); see [2-4].

However, the development of a mathematical apparatus for irregular (hyperbolic) dynamics comes from mathematicians and theoretical physicists of the Russian school; see the review paper [5] and the reference cited therein. Moreover the development of high speed computers has also allowed for displaing the complex behavior of the solutions visually.

Nowadays, nonlinear dynamics can be found in almost every branch of the applied science. It includes systems in which feedback, iterations, nonlinear interactions, and the general dependency of each part of the system upon the behavior of all other parts demand the use of nonlinear differential equations rather than the well-known linear differential equations, for example, Bellman equation [6] (with applications in economics [7]), Boltzmann equation [8] (with applications to gas dynamics), Colebrook equation [9] (with applications to turbulence), Ginzburg-Landau equation [10] (with applications to superconductors), NavierStokes equation [11] (with applications to fluid dynamics), Korteweg-De Vries equation [12] (for models of waves on shallow water surfaces), Sine-Gordon equation [13] (with applications to the study of crystal dislocations), LandauLifshitz-Gilbert equation [14] (with application to the precessional motion of magnetization in a solid), Ishimori equation [15], Vlasov equation [16] (with applications in plasma), nonlinear Schrodinger equation [17] (with applications to optics and water waves), Lienard equation [18] (with applications to oscillating circuits), Solow equation [19] (with applications to the economy), and Cournot-Bertrand equation [20] (with applications to the economy), Matsumoto-Nonaka equation [21] (with applications to the economy), Kaleckian equation [22] (with applications to the economy), Dullin-GottwaldHolm equation [23] (with applications to the propagation of surface waves in a shallow water regime), LotkaVolterra equation [24] (with applications in biology and economics), and thermostatted kinetic equations (with applications to physics, biology, vehicular traffic, crowds and swarms dynamics, and social and economic systems); see papers [25-34] and the review [35].

The motions involved in nonlinear equations are not simply combinations of a bunch of simpler motions. Moreover the dynamics involving nonlinear (ordinary, partial, or 
integro) differential equations are extremely different, and the related mathematical methods and analysis are problem dependent. Numerical simulations are also carried out for supporting the results.

The qualitative analysis of nonlinear ordinary differential equations is usually performed by searching conserved quantities (an approach that is typically used in Hamiltonian systems) and/or dissipative quantities. Linearization of the equations by Taylor expansion, change of variables, bifurcation theory, and perturbation methods is the most used approaches.

Nonlinear partial differential equations are qualitatively analyzed by using change the variables, separation of variables, and integral transforms. Other methods include the examination of the characteristics curves, and scale analysis (typically in fluid and heat mechanics) that allows, in some cases, for simplifing the nonlinear Navier-Stokes equations.

The nonlinear dynamics that appear in the above mentioned equations, and in the papers of this special issues, include highly sensitive to initial conditions (chaos dynamics, see; among others, papers [36-38]); anomalous transport (see paper [39] and references cited therein); alternating between two or more exclusive states (multistability; see [40, 41]); aperiodic oscillations (known as chaotic oscillations; see papers $[42,43]$ and the references section); amplitude death (complete cessation of oscillations; see [44, 45]); solitons (selfreinforcing solitary wave; see [46]); bifurcations (changes in the qualitative or topological property).

In this special issue, the tools of nonlinear dynamics have been used in attempts to better understand irregularity in diverse mathematical models of population dynamics, physics, biology, and economy. The interested reader is addressed to explore these interesting and fascinating results further. Moreover applications can refer to more research fields.

The guest editors of this special issue hope that problems discussed and investigated in the papers by the authors of this issue can inspire and motivate researchers in these fields to discover new, innovative, and novel applications in all areas of pure and applied mathematics.

\section{Acknowledgments}

The guest editors would like to express their gratitude to the reviewers for their serious evaluation of the papers submitted in this special issue. Thanks also go to the authors for their interesting papers. Carlo Bianca acknowledges the support by the FIRB project RBID08PP3J (Metodi matematici e relativi strumenti per la modellizzazione e la simulazione della formazione di tumori, competizione con il sistema immunitario, e conseguenti suggerimenti terapeutici).

Luca Guerrini

Carlo Bianca

Massimiliano Ferrara Constantin Udriste

\section{References}

[1] I. Newton, Philosophice Naturalis Principia Mathematica, Cambridge Digital Library, Cambridge, Mass, USA, 1687.

[2] N. D. Hulkower, “The zero energy three body problem," Indiana University Mathematics Journal, vol. 27, no. 3, pp. 409-447, 1978.

[3] N. D. Hulkower, "Central configurations and hyperbolic-elliptic motion in the three-body problem," Celestial Mechanics, vol. 21, pp. 37-41, 1980.

[4] S. J. Aarseth, Gravitational n-Body Simulations, Cambridge University Press, Cambridge, UK, 2003.

[5] C. Bianca, "On the mathematical transport theory in microporous media: the billiard approach," Nonlinear Analysis: Hybrid Systems, vol. 4, no. 4, pp. 699-735, 2010.

[6] R. Bellman, Dynamic Programming, Princeton University Press, Princeton, NJ, USA, 1957.

[7] M. Beckmann and R. Muth, "On the solution to the fundamental equation of inventory theory," Cowles Commission Discussion Paper 2116, 1954.

[8] R. J. DiPerna and P.-L. Lions, "On the Cauchy problem for Boltzmann equations: global existence and weak stability," Annals of Mathematics, vol. 130, no. 2, pp. 321-366, 1989.

[9] C. F. Colebrook, "Turbulent flow in pipes, with particular reference to the transition region between smooth and rough pipe laws," Journal of the Institution of Civil Engineers, vol. 11, pp. 133-156, 1939.

[10] L. D. Landau, Collected Papers, Pergamon Press, Oxford, UK, 1965.

[11] G. K. Batchelor, An Introduction to Fluid Dynamics, Cambridge University Press, Cambridge, UK, 1967.

[12] J. W. Miles, "The Korteweg-De Vries equation: a historical essay," Journal of Fluid Mechanics, vol. 106, pp. 131-147, 1981.

[13] J. Rubinsten, "Sine-Gordon equation," Journal of Mathematical Physics, vol. 11, pp. 258-266, 1970.

[14] I. Cimrák, "A survey on the numerics and computations for the Landau-Lifshitz equation of micromagnetism," Archives of Computational Methods in Engineering, vol. 15, no. 3, pp. 1-37, 2007.

[15] Y. Ishimori, "Multivortex solutions of a two-dimensional nonlinear wave equation," Progress of Theoretical Physics, vol. 72, no. 1, pp. 33-37, 1984.

[16] A. A. Vlasov, Many-Particle Theory and Its Application to Plasma, Gordon and Breach, New York, NY, USA, 1961.

[17] V. E. Zakharov and S. V. Manakov, "On the complete integrability of a nonlinear Schrödinger equation," Theoretical and Mathematical Physics, vol. 19, no. 3, pp. 551-559, 1974.

[18] A. Lienard, "Etude des oscillations entretenues," Revue générale de l'électricité, vol. 23, pp. 901-912, 1928.

[19] R. M. Solow, "A contribution to the theory of economic growth," Quarterly Journal of Economics, vol. 70, pp. 65-94, 1956.

[20] C. H. Tremblay and V. J. Tremblay, "The Cournot-Bertrand model and the degree of product differentiation," Economics Letters, vol. 111, no. 3, pp. 233-235, 2011.

[21] A. Matsumoto and Y. Nonaka, "Statistical dynamics in a chaotic Cournot model with complementary goods," Journal of Economic Behavior and Organization, vol. 61, no. 4, pp. 769-783, 2006.

[22] H. R. Dullin, G. A. Gottwald, and D. D. Holm, "An integrable shallow water equation with linear and nonlinear dispersion," Physical Review Letters, vol. 87, pp. 4501-4504, 2001. 
[23] M. Kalecki, "A macrodynamic theory of business cycles," Econometrica, vol. 3, pp. 327-344, 1935.

[24] J. D. Murray, Mathematical Biology I: An Introduction, Springer, New York, NY, USA, 2003.

[25] P. Degond and B. Wennberg, "Mass and energy balance laws derived from high-field limits of thermostatted Boltzmann equations," Communications in Mathematical Sciences, vol. 5, no. 2, pp. 355-382, 2007.

[26] B. Wennberg and Y. Wondmagegne, "The Kac equation with a thermostatted force field," Journal of Statistical Physics, vol. 124, no. 2-4, pp. 859-880, 2006.

[27] B. Wennberg and Y. Wondmagegne, "Stationary states for the Kac equation with a Gaussian thermostat," Nonlinearity, vol. 17, no. 2, pp. 633-648, 2004.

[28] V. Bagland, "Well-posedness and large time behaviour for the non-cutoff Kac equation with a Gaussian thermostat," Journal of Statistical Physics, vol. 138, no. 4-5, pp. 838-875, 2010.

[29] V. Bagland, B. Wennberg, and Y. Wondmagegne, "Stationary states for the noncutoff Kac equation with a Gaussian thermostat," Nonlinearity, vol. 20, no. 3, pp. 583-604, 2007.

[30] C. Bianca, "Modeling complex systems by functional subsystems representation and thermostatted-KTAP methods," Applied Mathematics and Information Sciences, vol. 6, pp. 495499, 2012.

[31] C. Bianca, "An existence and uniqueness theorem to the Cauchy problem for thermostatted-KTAP models," International Journal of Mathematical Analysis, vol. 6, no. 17-20, pp. 813-824, 2012.

[32] C. Bianca, "Onset of nonlinearity in thermostatted active particles models for complex systems," Nonlinear Analysis: Real World Applications, vol. 13, no. 6, pp. 2593-2608, 2012.

[33] C. Bianca, "Existence of stationary solutions in kinetic models with Gaussian thermostats," Mathematical Methods in the Applied Sciences., vol. 36, pp. 1768-1775, 2013.

[34] C. Bianca, M. Ferrara, and L. Guerrini, "High-order moments conservation in thermostatted kinetic models," Journal of Global Optimization, 2013.

[35] C. Bianca, "Thermostatted kinetic equations as models for complex systems in physics and life sciences," Physics of Life Reviews, vol. 9, pp. 359-399, 2012.

[36] S. H. Kellert, In the Wake of Chaos: Unpredictable Order in Dynamical Systems, University of Chicago Press, Chicago, Ill, USA, 1993.

[37] V. G. Ivancevic and T. T. Ivancevic, Complex Nonlinearity: Chaos, Phase Transitions, Topology Change, and Path Integrals, Springer, Berlin, Germany, 2008.

[38] C. Bianca and L. Rondoni, "The nonequilibrium Ehrenfest gas: a chaotic model with flat obstacles?” Chaos, vol. 19, no. 1, Article ID 013121, 10 pages, 2009.

[39] O. G. Jepps, C. Bianca, and L. Rondoni, "Onset of diffusive behavior in confined transport systems," Chaos, vol. 18, no. 1, Article ID 013127, 13 pages, 2008.

[40] D. Angeli, "Multistability in systems with counter-clockwise input-output dynamics," IEEE Transactions on Automatic Control, vol. 52, no. 4, pp. 596-609, 2007.

[41] F. Freyer, J. A. Roberts, P. Ritter, and M. Breakspear, "A canonical model of multistability and scale-invariance in biological systems," PLoS Computational Biology, vol. 8, no. 8, Article ID e1002634, 15 pages, 2012.

[42] F. G. Zvereva and O. S. Shcheglov, "Investigation of aperiodic oscillations in the plasma of a discharge under a low pressure,"
Radiophysics and Quantum Electronics, vol. 17, no. 11, pp. 13161319, 1974.

[43] H. Tanaka and T. Ushio, "Analysis of aperiodic oscillations in a flow model of a switching system," International Journal of Bifurcation and Chaos in Applied Sciences and Engineering, vol. 13, no. 4, pp. 981-994, 2003.

[44] V. Resmi, G. Ambika, and R. E. Amritkar, "General mechanism for amplitude death in coupled systems," Physical Review E, vol. 84, no. 4, Article ID 046212, 2011.

[45] V. Resmi, G. Ambika, R. E. Amritkar, and G. Rangarajan, "Amplitude death in complex networks induced by environment," Physical Review E, vol. 85, no. 4, Article ID 046211, 2012.

[46] M. Dunajski, Solitons, Instantons, and Twistors, Oxford University Press, Oxford, UK, 2009. 


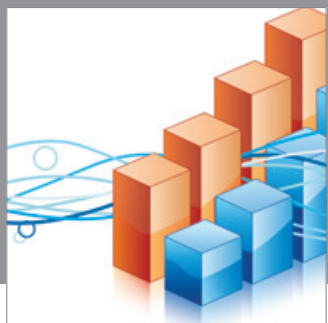

Advances in

Operations Research

mansans

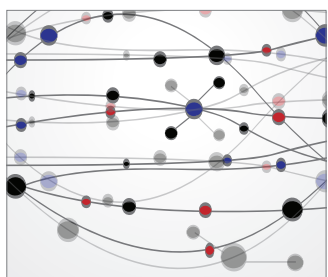

The Scientific World Journal
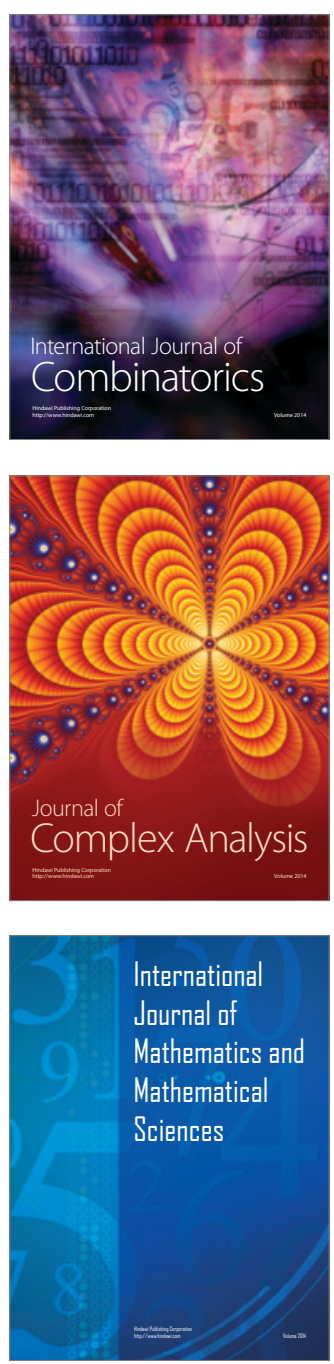
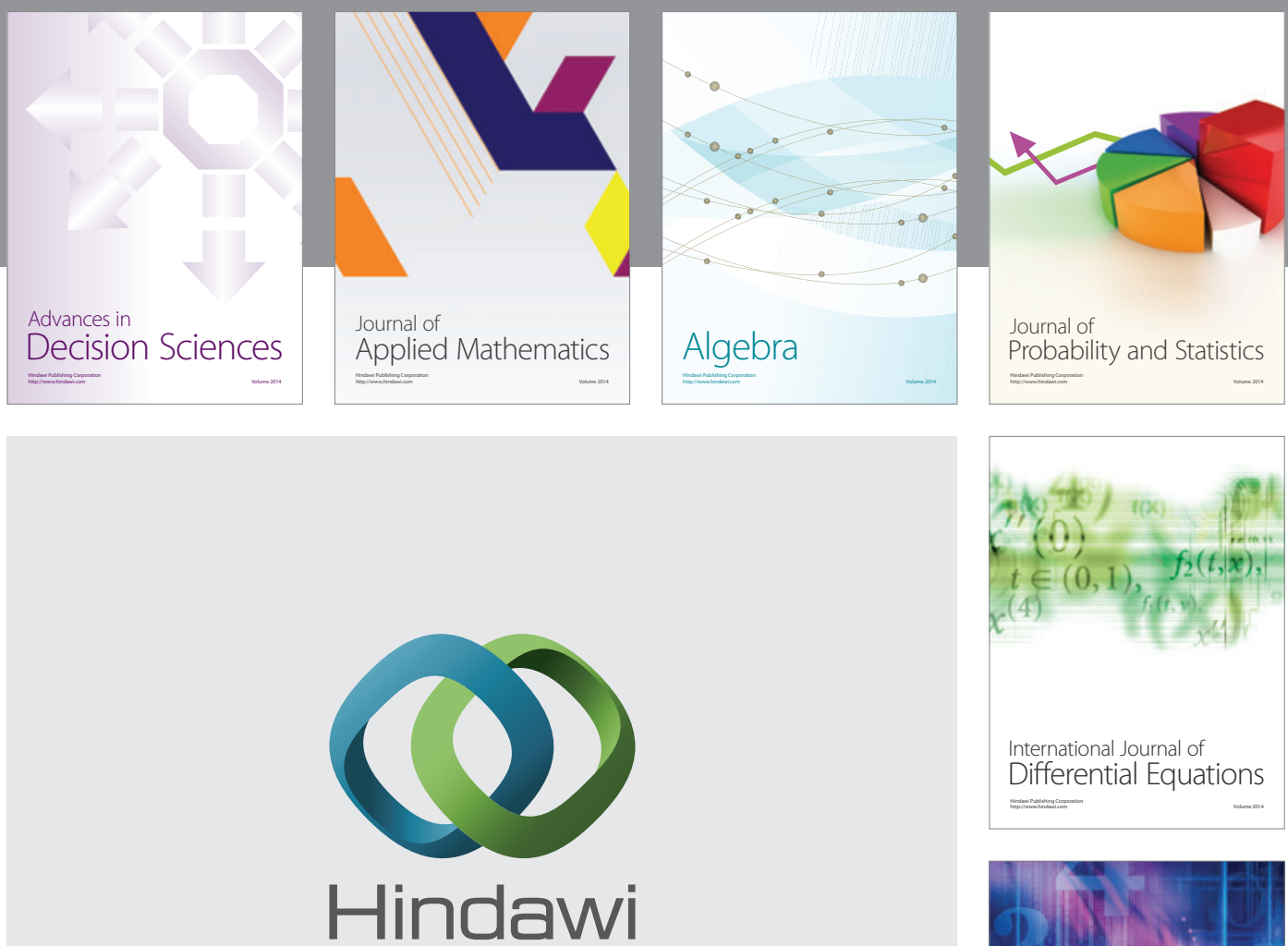

Submit your manuscripts at http://www.hindawi.com
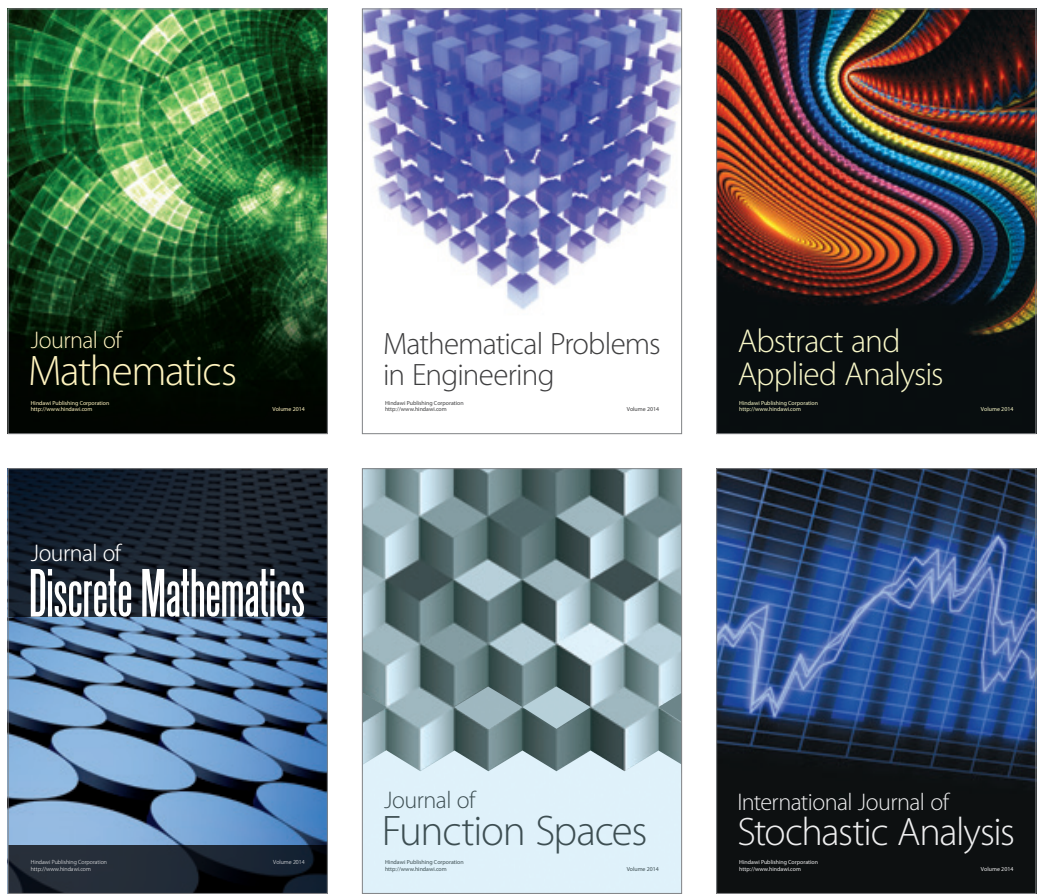

Journal of

Function Spaces

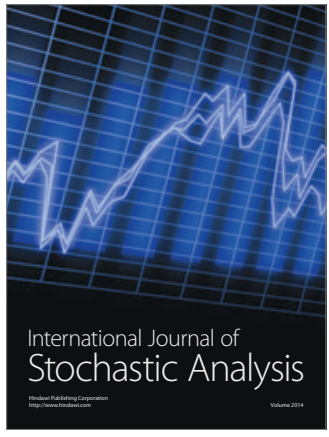

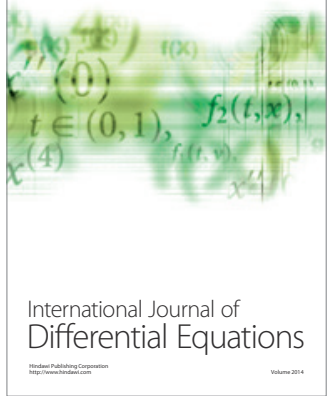
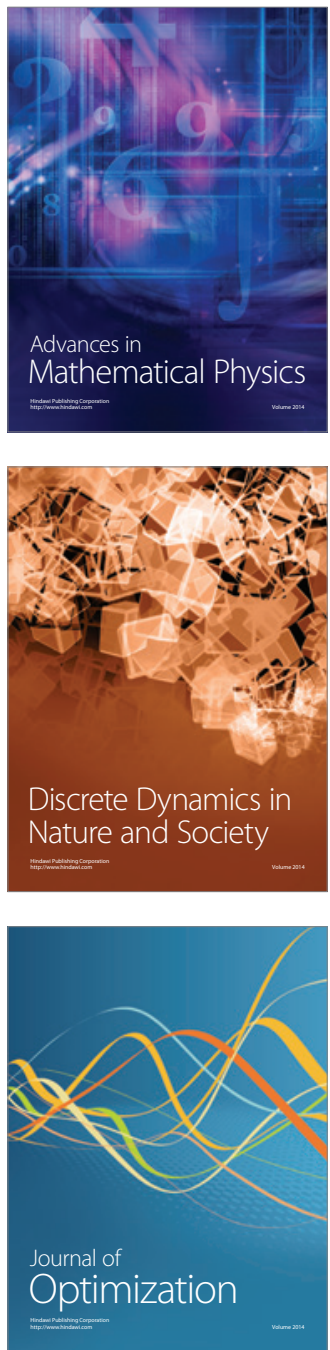